\title{
A 44K microarray dataset of the changing transcriptome in developing Atlantic salmon (Salmo salar L.)
}

\author{
Stuart G Jantzen ${ }^{\dagger}$, Dan S Sanderson ${ }^{\dagger}$, Kris R von Schalburg, Motoshige Yasuike, Francesco Marass and Ben F Koop ${ }^{*}$
}

\begin{abstract}
Background: Atlantic salmon (Salmo salar L.) is an environmentally and economically important organism and its gene content is reasonably well characterized. From a transcriptional standpoint, it is important to characterize the changes in gene expression over the course of unperturbed early development, from fertilization through to the parr stage.

Findings: S. salar samples were taken at 17 time points from 2 to 89 days post fertilization. Total RNA was extracted and CRNA was synthesized and hybridized to a newly developed 44K oligo salmonid microarray platform. Quantified results were subjected to preliminary data analysis and submitted to NCBI's Gene Expression Omnibus (GEO). Data can be found under the GEO accession number GSE25938. http://www.ncbi.nlm.nih.gov/geo/query/acc. cgi?acc $=$ GSE25938

Conclusions: Throughout the entire period of development, several thousand genes were found to be differentially regulated. This work represents the trancriptional characterization of a very large geneset that will be extremely valuable in further examination of the transcriptional changes in Atlantic salmon during the first few months of development. The expression profiles can help to annotate salmon genes in addition to being used as references against any number of experimental variables to which developing salmonids might be subjected.
\end{abstract}

\section{Background}

Atlantic salmon (Salmo salar L.) are an environmentally and economically important organism. The genome has been well studied and is currently being fully sequenced [1-4]. In addition, a number of microarrays have been developed for transcription studies of S. salar [2,5-7]. As a benefit of the extensive characterization of the transcriptome of $S$. salar, large scale studies of gene expression changes can be undertaken using these microarray platforms [8].

This study is the first to utilize a newly developed $44 \mathrm{~K}$ oligo salmonid microarray design, one of the first salmonid oligo microarrays. This array comprises approximately 22,000 60-mer oligos that were conserved (95\% similar) between rainbow trout (Oncorhynchus mykiss) and Atlantic salmon [6] plus 14,866 additional Atlantic

\footnotetext{
* Correspondence: bkoop@uvic.ca

+ Contributed equally

Center for Biomedical Research, University of Victoria, Victoria, British Columbia, V8W 3N5, Canada
}

salmon and 5,661 additional rainbow trout contig sequences. The result is a microarray that has a large transcript representation with very low redundancy. The array is composed of oligos based on roughly $80 \%$ S. salar and $20 \%$ O. mykiss contigs. $84 \%$ of all features are well annotated with fairly stringent hits (e-value cutoff: 1e-10) to public databases (December 17, 2009). The annotation files may be found at the cGRASP microarray page [9]. Efforts to annotate unknown contigs will continue.

Library construction, sequence analysis and contig assembly have been described previously [2]. The 14,866 additional $S$. salar oligos were all derived from selected contigs compiled in the local database of the authors (August 11, 2009) [10]. These were chosen first from the approximately 10,000 full length cDNAs in the database [11]. The remainder were selected from well annotated sequences and then from poorly annotated sequences with an open reading frame longer than 300 bp represented by two or more clones. 5,206 of the
C Biomed Central

() 2011 Koop et al; licensee BioMed Central Ltd. This is an open access article distributed under the terms of the Creative Commons Attribution License (http://creativecommons.org/licenses/by/2.0), which permits unrestricted use, distribution, and reproduction in any medium, provided the original work is properly cited. 
additional O. mykiss contig-derived oligos were selected from the set of well annotated sequences in the local database that did not have a clear homologous representative in S. salar. The remaining 455 were selected from annotated NCBI Nucleotide resources (July 21, 2009) [12] with priority given to immune system related sequences. Representative oligos from sequences identified by Gene Ontology (GO) [13] were included. After sequence selection, oligos were derived from the selected contigs by Agilent Technologies (Santa Clara, $\mathrm{CA}$ ) and were $60 \mathrm{bp}$ in length. The oligo selection process was biased in favor of 3' sequences. While the majority of oligos are unique to contigs (i.e. only one spot on the array can be mapped back to a given contig), approximately $27 \%$ of oligos, including the original 22,000 , were derived from the same contig as at least one other oligo. Finally, in situ oligo synthesis and microarray manufacturing was performed by Agilent. Microarray slides are available through Agilent's eArray platform, with each slide containing four arrays [14].

Due to the very large number of unique features on this platform, a genome-wide exploration of expression levels in salmonids is expected to produce significant and detailed information on many molecular systems. For example, the genetic factors involved in the very early developmental stages of Atlantic salmon are not completely understood. It is therefore of interest to do a thorough examination of $S$. salar developmental stages from fertilization through to the parr stage, using a transcriptomics approach. Recently, another group has used a microarray platform to profile the changes in gene expression during smoltification in Atlantic salmon [15] when freshwater parr make the transition to saltwater smolt. The dataset presented here complements this earlier study.

The objective was to comprehensively monitor the salmonid transcriptome during controlled and unperturbed development. This work complements and facilitates recent efforts to sequence and annotate the Atlantic salmon genome. It further provides a resource that identifies expression levels of tens of thousands of genes during the course of development. These baseline expression patterns can be used as references in future experiments to examine physiological, reproductive, mutational, and environmental variables.

\section{Materials and methods}

\section{Animals and sampling}

Treatment of the fish used in this study was in compliance with the regulations of the University of Victoria Animal Care Committee. Eggs from Atlantic salmon (McConnell (Mowi)) were obtained in November, 2009 from Marine Harvest United Hatchery (Fanny Bay, BC, Canada). The eggs were fertilized by gently mixing the eggs and milt by hand and then washed with partial exchanges of water. Approximately 2,000 fertilized eggs were then transferred and placed in Heath trays (Marisource, Fife, WA) at the University of Victoria. The embryos and larvae were raised in fresh water at a temperature of $12^{\circ} \mathrm{C}$ and a flow rate of 200 liters/h.

The day of fertilization was marked as 0 days post fertilization (dpf), and hatching and yolk sac absorption occurred between 38 to $40 \mathrm{dpf}$ and 68 to $70 \mathrm{dpf}$, respectively. Whole embryos $(n=20)$ and larvae were collected every one to three days for several weeks. Alevin and fry were collected every sixth day for the remainder of the study (Figure 1). Unfertilized eggs were not included in the experimental design, which could be used to examine possible RNA effects from the oocyte. Samples were directly placed into dry ice and stored at $-80^{\circ} \mathrm{C}$ until RNA extraction.

\section{RNA extraction}

Total RNAs were extracted in TRIzol reagent (Invitrogen, Carlsbad, CA) by mixer-mill homogenization (Retsch, Newtown, PA) and spin-column purified using RNeasy Mini kits (Qiagen, Hilden, Germany). The RNA was extracted from three whole individual embryos, larvae, or alevins at $2 \mathrm{dpf}$ and then every fifth day (5 to $35 \mathrm{dpf}$ ) or sixth day (35 to $89 \mathrm{dpf}$ ). Quantity and quality of RNA samples were then measured using UV absorbance (NanoDrop Technologies, Wilmington, DE) and quality was also checked by agarose gel electrophoresis.

\section{cRNA synthesis, labeling, and purification}

Cy5 labeled experimental cRNA samples were generated using an Agilent Low Input Quick Amp (LIQA) kit, following the manufacturer's instructions. For each time point, $40 \mathrm{ng}$ of total RNA from three individuals was used to generate first-strand cDNA. Agilent Spike-In B control RNA was included in each reaction. After the denaturation step $\left(10 \mathrm{~min}\right.$ at $\left.65^{\circ} \mathrm{C}\right)$ and cRNA synthesis step $\left(2 \mathrm{hr}\right.$ at $\left.40^{\circ} \mathrm{C}\right)$, the reactions were incubated at $70^{\circ} \mathrm{C}$ for 15 minutes to inactivate the AffinityScript enzyme and subsequently stored at $-80^{\circ} \mathrm{C}$ until further use. For the labeling reactions, thawed cRNA samples were each mixed with $16 \mu \mathrm{L}$ of Transcription Master Mix cocktail containing Cy5 dye, and incubated at $40^{\circ} \mathrm{C}$ for two hours. Purification was performed using Qiagen RNeasy mini spin columns, eluting in $30 \mu \mathrm{L}$ of RNase-free water. For the generation of the reference pool, equimolar amounts from the three individuals in each time point were pooled to give $120 \mathrm{ng}$ of total RNA used in each first-strand reaction. Spike-In A control RNA was included in each reaction. After labeling with $\mathrm{Cy} 3$ and column purification as above, a common reference pool was created by including $2.8 \mu \mathrm{g}$ of cRNA from each time point, except for $2 \mathrm{dpf}$, for which only $1.3 \mu \mathrm{g}$ of 


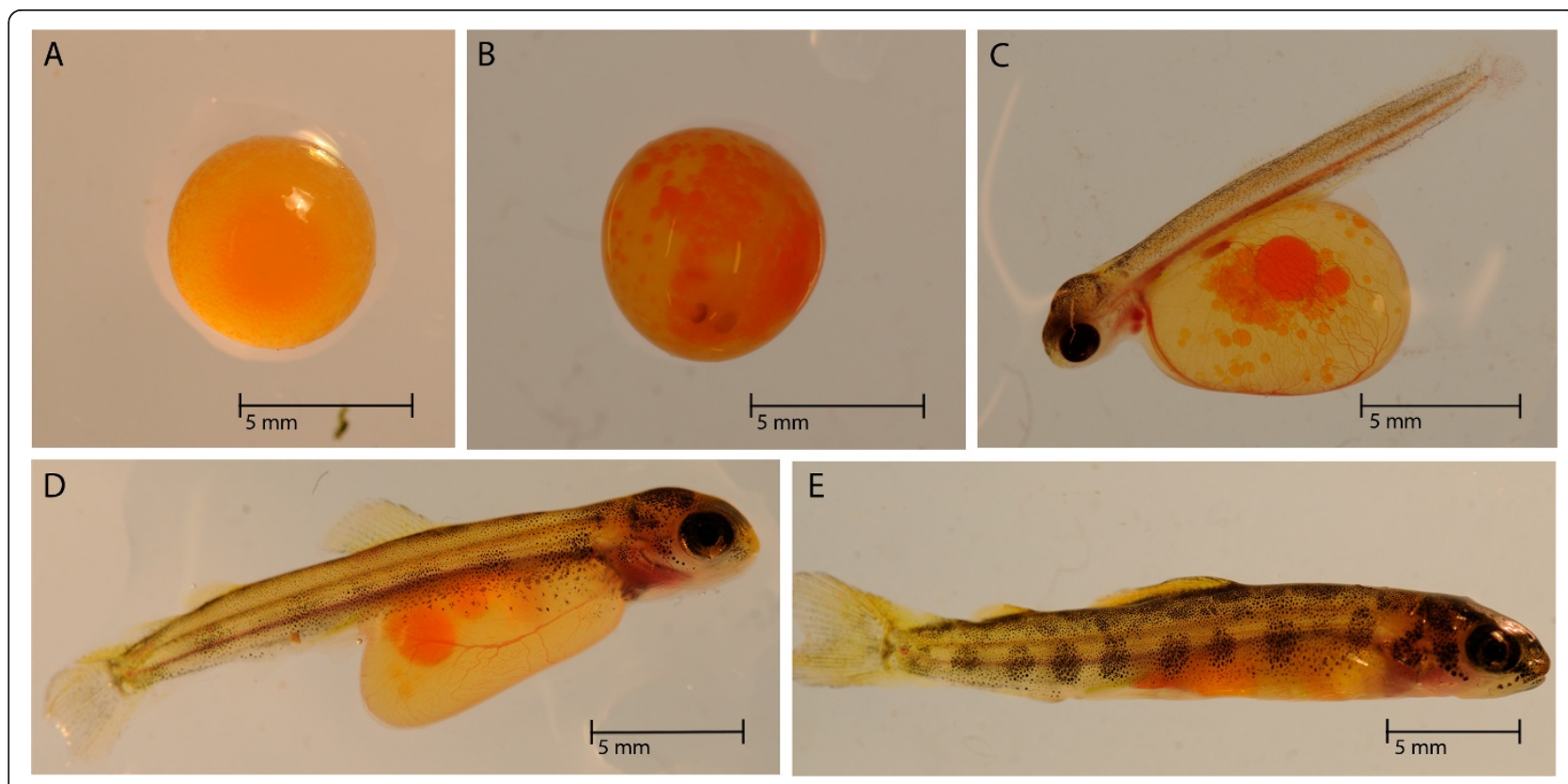

Figure 1 S. salar during development. Photographs taken during development of Atlantic salmon at the University of Victoria. A) 5 B) 20 C) 41 D) 59 E) $77 \mathrm{dpf}$.

material was ultimately available. Due to the small size and early developmental stage of samples from days 2 , 5 , and $10 \mathrm{dpf}$, limited RNA quantities necessitated additional extractions and subsequent synthesis and labeling reactions, however repeated procedures produced $\mathrm{Cy} 5$ labeled cRNA of the required quantity and quality.

\section{Microarray hybridization and scanning}

Experimental samples of Cy5 labeled cRNA were quantified on a Nanodrop ND-1000. All samples were found to be of sufficient specific activity with a mean $( \pm$ SD) of $18.22 \pm 2.03 \mathrm{pmol} \mathrm{Cy} 5 / \mu \mathrm{g}$ cRNA as per manufacturer's recommendation (Agilent) and an appropriate RNA absorbance ratio with a mean of $2.29 \pm 0.06$. Next, cRNA fragmentation mixtures were created following the LIQA kit instructions, using $825 \mathrm{ng}$ of experimental sample and $825 \mathrm{ng}$ of reference pool. These mixtures were incubated at $60^{\circ} \mathrm{C}$ for 30 minutes. After cooling on ice for one minute, hybridization mixtures were prepared by adding 2x GEx Hybridization Buffer HI-RPM and mixing well by pipetting. These reactions were loaded in random arrangements with respect to time point onto $44 \mathrm{~K}$ oligo salmonid microarrays (Agilent025055) using Agilent SureHyb Hybridization Chambers. Each of the $4 \times 44 \mathrm{~K}$ arrays on the microarray slides had $100 \mu \mathrm{L}$ of hybridization reaction added. The hybridization reactions were allowed to occur for 17 hours at $65^{\circ} \mathrm{C}$. Slide washes were performed as per the manufacturer's instructions, including an ozone-protection step using the Agilent Stabilization and Drying
Solution. Slides were scanned as soon as possible on a ScanArray Express (PerkinElmer, Waltham, MA) scanner at $5 \mu \mathrm{m}$ resolution using a PMT setting of 80 in both channels, a black threshold of 1800 , and a full color threshold of 26.8. Slides were stored in a low ozone chamber (typically $<5 \mathrm{ppb}$ ) until scanned.

\section{Data processing}

Since the temperature of the environment has a strong influence on the rate of development of Atlantic salmon [16], time points as dpf were converted to both degree days and to the relative age in terms of Tau-somite $\left(\tau_{\mathrm{s}}\right)$ as proposed by Gorodilov [17] (Table 1). This allowed for the determination of corresponding phenotypic stages independent of the temperature during development.

This study complies with the MIAME standards [18]. Scanned arrays were quantified using Imagene 8.0 (Biodiscovery, El Segundo, CA) and processed with in-house scripts for input to GeneSpring GX 11.0 (Agilent). Data were imported into GeneSpring under the following conditions: raw data were converted to a threshold value of 1.0, data were log-transformed, a Lowess normalization was performed, and a baseline transformation to the median of all samples was performed. As a quality control measure, 3D-PCA graphs were examined with respect to the experimental variable, namely days post fertilization (Figure 2a), and technical variables such as slide number (Figure $2 b$ ), with four randomized samples per slide. Clustering according to days post fertilization was evident, with earlier days $(2,5$, and 10$)$ 
Table 1 S. salar developmental stages sampled

\begin{tabular}{|c|c|c|c|}
\hline Days post fertilization (dpf) & Degree days $\left(12^{\circ} \mathrm{C} * \mathrm{dpf}\right)$ & Relative age in $\tau_{\mathrm{s}}$ & Subperiod \\
\hline 2 & 24 & 17 & Blastulation \\
\hline 5 & 60 & 42 & Gastrulation \\
\hline 10 & 120 & 83 & Somitogenesis \\
\hline 15 & 180 & 125 & Vascularization of yolksac \\
\hline 20 & 240 & 167 & Vascularization of yolksac \\
\hline 25 & 300 & 208 & Formation of caudal rays \\
\hline 30 & 360 & 250 & Formation of caudal rays \\
\hline 35 & 420 & 292 & Formation of caudal rays \\
\hline 41 & 492 & 342 & Free embryo/Alevin \\
\hline 47 & 564 & 392 & Free embryo/Alevin \\
\hline 53 & 636 & 442 & Free embryo/Alevin \\
\hline 59 & 708 & 492 & $\begin{array}{l}\text { Alevins have left gravel/ } \\
\text { Beginning of parr markings }\end{array}$ \\
\hline 65 & 780 & 542 & Fry/Appearance of caudal parr marks \\
\hline 71 & 852 & 592 & Fry/Yolk-sac completely absorbed \\
\hline 77 & 924 & 642 & Parr \\
\hline 83 & 996 & 692 & Parr \\
\hline 89 & 1068 & 742 & Parr \\
\hline
\end{tabular}

S. salar samples were taken at 17 time points, which correspond to specific developmental stages depending on the environmental temperature. Developmental stage can be measured in degree days, as relative age in Tau-somite $\left(\tau_{s}\right)$ [16], or described as a phenotypic subperiod as expected in the wild [16].

being further removed from the rest of the samples. In contrast, no real clustering was observable based on slide number, and this trend continued for other technical variables such as labelling day (data not shown). After data import, entities flagged for various reasons (e.g. poor spot morphology) by Imagene were filtered out, along with entities possessing raw signal values lower than 500 .

As an initial exploratory analysis, conditions (dpf) were compared to each other using a sliding window approach. Using a t-test assuming unequal variance (Welch) ( $\mathrm{p} \leq 0.01$, fold change $\geq 2.0$ ) without multiple

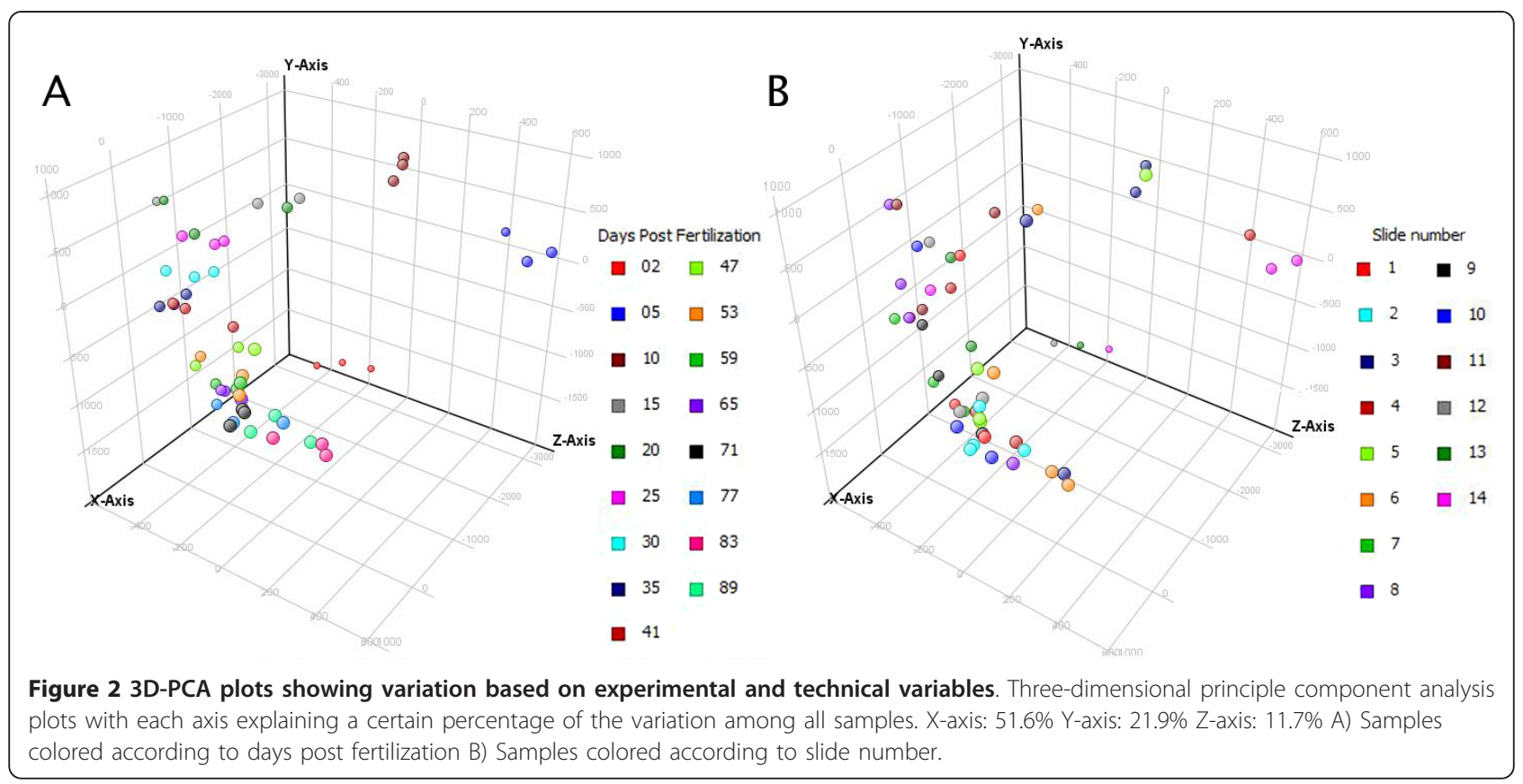


Table 2 Numbers of differentially regulated entities across timeline

\begin{tabular}{ll}
\hline Comparison (dpf) & Number of differentially regulated entities \\
\hline 2 vs. 5 & 3329 \\
\hline 5 vs. 10 & 2325 \\
\hline 10 vs. 15 & 804 \\
\hline 15 vs. 20 & 102 \\
\hline 20 vs. 25 & 59 \\
\hline 25 vs. 30 & 62 \\
\hline 30 vs. 35 & 127 \\
\hline 35 vs. 41 & 53 \\
\hline 41 vs. 47 & 47 \\
\hline 47 vs. 53 & 51 \\
\hline 53 vs. 59 & 38 \\
\hline 59 vs. 65 & 54 \\
\hline 65 vs. 71 & 22 \\
\hline 71 vs. 77 & 12 \\
\hline 77 vs. 83 & 68 \\
\hline 83 vs. 89 & 84 \\
\hline A Students t-st assum
\end{tabular}

A Student's t-test assuming unequal variation ( $P \leq 0.01, F C \geq 2.0$ ) without MTC was used to compare each sampled time point to the subsequent time point. Numbers of entities that were significantly differentially expressed between the compared time points are presented.

test correction (MTC), each time point was compared to the next time point in the series. This allowed a cursory examination of the genes that were differentially regulated as development progressed. Numbers of significantly differentially expressed entities are listed in Table 2 and lists of annotated entities are provided as supplementary tables (additional file 1, additional file 2, additional file 3 , additional file 4 , additional file 5 , additional file 6 , additional file 7 , additional file 8 , additional file 9 , additional file 10 , additional file 11 , additional file 12 , additional file 13, additional file 14, additional file 15 , additional file 16). With a sample size of three individuals per condition, it is possible that discovery of more subtly changing differential regulation is limited and this may also cause inclusion of some false positives. Nonetheless, it is evident that high numbers of genes are being differentially regulated, especially in the first few weeks of development. In fact, it appears that the vast majority of transcriptional changes occur in the first 10 days of development.

In order to investigate the biological and technical variation within and among conditions, we examined the variation among biological replicates for each entity (Table 3). All 23,854 entities that passed pre-filtering were used to determine the average standard deviation of replicates. The first condition ( $2 \mathrm{dpf}$ ) has the highest mean, indicating a possibly higher level of noise due to biological and technical variation; however the median is highest at $15 \mathrm{dpf}$, and in fact all days have comparable
Table 3 Variation among replicate samples across timeline

\begin{tabular}{llll}
\hline Condition (dpf) & Mean & Median & Standard deviation \\
\hline 2 & 0.574 & 0.453 & 0.484 \\
\hline 5 & 0.412 & 0.334 & 0.359 \\
\hline 10 & 0.342 & 0.279 & 0.282 \\
\hline 15 & 0.511 & 0.459 & 0.327 \\
\hline 25 & 0.516 & 0.449 & 0.344 \\
\hline 30 & 0.408 & 0.354 & 0.285 \\
\hline 35 & 0.495 & 0.438 & 0.320 \\
\hline 41 & 0.439 & 0.369 & 0.339 \\
\hline 53 & 0.473 & 0.421 & 0.310 \\
\hline 59 & 0.440 & 0.370 & 0.326 \\
\hline 65 & 0.422 & 0.372 & 0.290 \\
\hline 71 & 0.351 & 0.288 & 0.272 \\
\hline 77 & 0.427 & 0.358 & 0.324 \\
\hline 83 & 0.416 & 0.354 & 0.311 \\
\hline 89 & 0.490 & 0.427 & 0.331 \\
\hline For & 0.437 & 0.382 & 0.303 \\
\hline
\end{tabular}

For each entity passing expression threshold and spot flag filters (total = 23,854), the standard deviation of the normalized, log-transformed values for the three replicates in a given condition was determined. For the calculated standard deviations within each condition, the mean deviation, median deviation, and standard deviation of the deviations were determined.

values. It appears that the data is not substantially noisier in the first days, therefore it is likely that the much higher number of differential transcripts in these comparisons is biologically accurate.

In terms of ontogenetically relevant probes on the $44 \mathrm{~K}$ microarray, over 900 entities are currently annotated with the GO term "development". More specifically, approximately 620 and 180 entities are annotated with the terms "system development" and "embryo development", respectively. In this experiment, the majority of entities in each of these categories was expressed above our threshold of 500 in at least one condition. Some of the GO terms that are significantly enriched in the various comparisons include "blastocyst development" between 2 and $5 \mathrm{dpf}$, "brain development" between 5 and $10 \mathrm{dpf}$, "organ development" and "induction of apoptosis" between 10 and $15 \mathrm{dpf}$, and "erythrocyte development" between 15 and $20 \mathrm{dpf}$, to name just a few. Other researchers may perform fuller and more detailed analyses in accordance with their own questions and hypotheses.

\section{Use of dataset}

Beyond this preliminary analysis, there is a wealth of information to be gained from these data and we have submitted all normalized and raw data to NCBI's Gene Expression Omnibus (GEO) [19] for others to examine. 
The data are accessible through GEO Series accession number GSE25938 http://www.ncbi.nlm.nih.gov/geo/ query/acc.cgi?acc $=$ GSE25938. This dataset encompasses variation among three individuals per condition and differences across 17 timepoints. It is evident that this microarray provides the ability to determine a detailed transcriptional basis of ontogeny and this experiment in particular contains a great deal of developmental information. In addition, these data could be used as a reference for perturbed or abnormal development in other studies, or for researchers to refer to when transcriptional patterns of specific genes are discovered in other young salmonids.

\section{Conclusion}

Here we present a large and novel dataset that represents an invaluable source of information on the transcriptional changes present in developing salmon. We believe these data will be of interest to many researchers in several fields, including aquaculture, genomics, and developmental and evolutionary biology. Both as an examination of healthy development on its own and as a reference for future studies, this set of expression profiles will prove to be valuable to the scientific community.

\section{Additional material}

Additional file 1: 02 vs. 05 annotated entities. Spreadsheet of all annotated significantly differentially regulated entities between 2 and $5 \mathrm{dpf}$. Includes p-values, fold change, direction of regulation, and annotation information.

Additional file 2: 05 vs. 10 annotated entities. Spreadsheet of all annotated significantly differentially regulated entities between 5 and $10 \mathrm{dpf}$. Includes p-values, fold change, direction of regulation, and annotation information.

Additional file 3: 10 vs. 15 annotated entities. Spreadsheet of all annotated significantly differentially regulated entities between 10 and $15 \mathrm{dpf}$. Includes p-values, fold change, direction of regulation, and annotation information.

Additional file 4: 15 vs. 20 annotated entities. Spreadsheet of all annotated significantly differentially regulated entities between 15 and $20 \mathrm{dpf}$. Includes p-values, fold change, direction of regulation, and annotation information.

Additional file 5: 20 vs. 25 annotated entities. Spreadsheet of all annotated significantly differentially regulated entities between 20 and $25 \mathrm{dpf}$. Includes p-values, fold change, direction of regulation, and annotation information.

Additional file 6: 25 vs. 30 annotated entities. Spreadsheet of all annotated significantly differentially regulated entities between 25 and $30 \mathrm{dpf}$. Includes p-values, fold change, direction of regulation, and annotation information.

Additional file 7: $\mathbf{3 0}$ vs. 35 annotated entities. Spreadsheet of all annotated significantly differentially regulated entities between 30 and $35 \mathrm{dpf}$. Includes p-values, fold change, direction of regulation, and annotation information.

Additional file 8: 35 vs. 41 annotated entities. Spreadsheet of all annotated significantly differentially regulated entities between 35 and
$41 \mathrm{dpf}$. Includes p-values, fold change, direction of regulation, and annotation information.

Additional file 9: 41 vs. 47 annotated entities. Spreadsheet of all annotated significantly differentially regulated entities between 41 and $47 \mathrm{dpf}$. Includes p-values, fold change, direction of regulation, and annotation information.

Additional file 10: 47 vs. 53 annotated entities. Spreadsheet of all annotated significantly differentially regulated entities between 47 and $53 \mathrm{dpf}$. Includes p-values, fold change, direction of regulation, and annotation information.

Additional file 11: 53 vs. 59 annotated entities. Spreadsheet of all annotated significantly differentially regulated entities between 53 and $59 \mathrm{dpf}$. Includes $\mathrm{p}$-values, fold change, direction of regulation, and annotation information.

Additional file 12: 59 vs. 65 annotated entities. Spreadsheet of all annotated significantly differentially regulated entities between 59 and $65 \mathrm{dpf}$. Includes p-values, fold change, direction of regulation, and annotation information.

Additional file 13: 65 vs. 71 annotated entities. Spreadsheet of all annotated significantly differentially regulated entities between 65 and $71 \mathrm{dpf}$. Includes p-values, fold change, direction of regulation, and annotation information.

Additional file 14: 71 vs. 77 annotated entities. Spreadsheet of all annotated significantly differentially regulated entities between 71 and $77 \mathrm{dpf}$. Includes p-values, fold change, direction of regulation, and annotation information.

Additional file 15: 77 vs. 83 annotated entities. Spreadsheet of all annotated significantly differentially regulated entities between 77 and $83 \mathrm{dpf}$. Includes p-values, fold change, direction of regulation, and annotation information.

Additional file 16: 83 vs. 89 annotated entities. Spreadsheet of all annotated significantly differentially regulated entities between 83 and $89 \mathrm{dpf}$. Includes p-values, fold change, direction of regulation, and annotation information.

\section{Acknowledgements}

This project was funded by the Natural Sciences and Engineering Research Council of Canada. Thanks to Marine Harvest for the generous donation of the Atlantic salmon eggs for this study. Thanks also to Brendon Campbell, Amy Hoare, and Brian Ringwood for egg transport and care of developing fish (Animal Care Services, University of Victoria).

\section{Authors' contributions}

SGJ performed data processing and analysis and drafted the manuscript. DSS ran microarrays and performed initial data processing. KRVS performed animal sampling and RNA extractions. MY performed additional animal sampling and assisted with microarray reactions. FM designed the 44K microarray. BFK conceived of the study. All authors read and approved the final manuscript.

\section{Competing interests}

The authors declare that they have no competing interests.

Received: 15 December 2010 Accepted: 29 March 2011 Published: 29 March 2011

\section{References}

1. Ng HSS, Artieri GC, Bosdet El, Chiu RR, Danzmann GR, Davidson SW, Ferguson MM, Fjell DC, Hoyheim B, Jones JMS, de Jong JP, Koop FB, Krzywinski IM, Lubieniecki K, Marra AM, Mitchell AL, Mathewson C, Osoegawa K, Parisotto ES, Phillips BR, Rise LM, von Schalburg RK, Schein EJ, Shin SH, Siddiqui A, Thorsen J, Wye N, Yang G, Zhu LB: A physical map of the genome of Atlantic salmon, Salmo salar. Genomics 2005, 86(4):396-404. 
2. Koop BF, von Schalburg KR, Leong J, Walker N, Lieph R, Cooper GA, Robb A, Beetz-Sargent M, Holt RA, Moore R, Brahmbhatt S, Rosner J, Rexroad CE, McGowan CR, Davidson WS: A salmonid EST genomic study: genes, duplications, phylogeny and microarrays. BMC Genomics 2008, 9:545.

3. Phillips RB, Keatley KA, Morasch MR, Ventura AB, Lubieniecki KP, Koop BF, Danzmann RG, Davidson WS: Assignment of Atlantic salmon (Salmo salar) linkage groups to specific chromosomes: Conservation of large syntenic blocks corresponding to whole chromosome arms in rainbow trout (Oncorhynchus mykiss). BMC Genet 2009, 10:46.

4. Davidson WS, Koop BF, Jones SJ, Iturra P, Vidal R, Maass A, Jonassen I, Lien S, Omholt SW: Sequencing the genome of the Atlantic salmon (Salmo salar). Genome Biol 2010, 11:403.

5. von Schalburg RK, Rise LM, Cooper AG, Brown DG, Gibbs RA, Nelson CC, Davidson SW, Koop FB: Fish and chips: Various methodologies demonstrate utility of a 16,006-gene salmonid microarray. BMC Genomics 2005, 6:126.

6. von Schalburg KR, Cooper GA, Leong J, Robb A, Lieph R, Rise ML, Davidson WS, Koop BF: Expansion of the genomics research on Atlantic salmon Salmo salar L. project (GRASP) microarray tools. J Fish Biol 2008, 72:2051-2070.

7. Taggart JB, Bron JE, Martin SAM, Seear PJ, Hoyheim B, Talbot R, Carmichael SN, Villeneuve LAN, Sweeney GE, Houlihan DF, Secombes CJ, Tocher DR, Teale AJ: A description of the origins, design and performance of the TRAITS-SGP Atlantic salmon Salmo salar L. CDNA microarray. J Fish Biol 2008, 72(9):2071-2094.

8. Miller KM, Maclean N: Teleost microarrays: development in a broad phylogenetic range reflecting diverse applications. J Fish Biol 2008, 72(9):2039-2050

9. cGRASP Microarray Page. [http://web.uvic.ca/grasp/microarray/].

10. cGRASP. [http://web.uvic.ca/grasp].

11. Leong SJ, Jantzen GS, von Schalburg RK, Cooper AG, Messmer MA, Liao YN, Munro S, Moore R, Holt AR, Jones JMS, Davidson SW, Koop FB: Salmo salar and Esox lucius full-length CDNA sequences reveal changes in evolutionary pressures on a post-tetraploidization genome. BMC Genomics 2010, 11(1):279.

12. NCBI. [http://www.ncbi.nlm.nih.gov].

13. Ashburner M, Ball AC, Blake AJ, Botstein D, Butler H, Cherry MJ, Davis PA, Dolinski K, Dwight SS, Eppig TJ, Harris AM, Hill PD, Issel-Tarver L, Kasarskis A, Lewis S, Matese CJ, Richardson EJ, Ringwald M, Rubin MG, Sherlock G, GO Consortium: Gene Ontology: tool for the unification of biology. Nat Genet 2000, 25(1):25-29.

14. Agilent Technologies eArray. [https://earray.chem.agilent.com/earray].

15. Seear PJ, Carmichael SN, Talbot R, Taggart JB, Bron JE, Sweeney GE: Differential Gene Expression During Smoltification of Atlantic Salmon (Salmo salar L.): a First Large-Scale Microarray Study. Mar Biotechnol 2010, 12(2):126-140

16. Hamor T, Garside TE: Developmental rates of embryos of Atlantic salmon, Salmo salar L., in response to various levels of temperature, dissolved oxygen, and water exchange. Can J Zool 1976, 54(11):1912-1917.

17. Gorodilov NY: Description of the early ontogeny of the Atlantic salmon, Salmo salar, with a novel system of interval (state) identification. Environ Biol Fish 1996, 47(2):109-127.

18. Brazma A, Hingamp P, Quackenbush J, Sherlock G, Spellman P, Stoeckert C, Aach J, Ansorge W, Ball AC, Causton CH, Gaasterl T, Glenisson P, Holstege CPF, Kim Fl, Markowitz V, Matese CJ, Parkinson H, Robinson A, Sarkans U, Schulze-Kremer S, Stewart J, Taylor R, Vilo J, Vingron M: Minimum information about a microarray experiment (MIAME) - toward standards for microarray data. Nat Genet 2001, 29(4):365-371.

19. Edgar R, Domrachev M, Lash EA: Gene Expression Omnibus: NCBI gene expression and hybridization array data repository. Nucleic Acids Res 2002, 30(1):207-210.

doi:10.1186/1756-0500-4-88

Cite this article as: Jantzen et al: A $44 \mathrm{~K}$ microarray dataset of the changing transcriptome in developing Atlantic salmon (Salmo salar L.). BMC Research Notes 2011 4:88.

\section{Submit your next manuscript to BioMed Central and take full advantage of:}

- Convenient online submission

- Thorough peer review

- No space constraints or color figure charges

- Immediate publication on acceptance

- Inclusion in PubMed, CAS, Scopus and Google Scholar

- Research which is freely available for redistribution

Submit your manuscript at www.biomedcentral.com/submit
C Biomed Central 